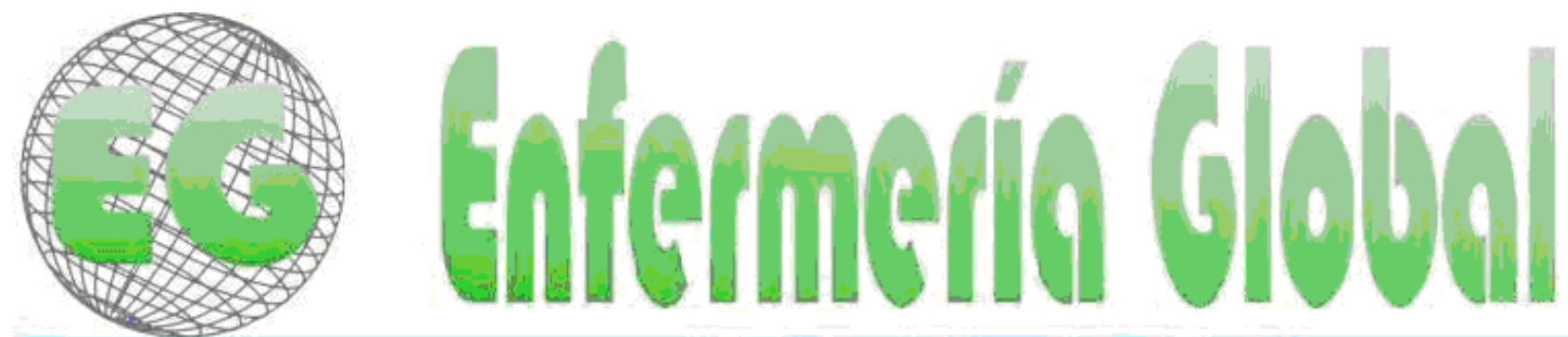

$\mathrm{N}^{\circ} 16$

Revista electrónica cuatrimestral de Enfermeria

Junio 2009

www.um.es/eglobal/

ADMINISTRAGIÓN - GESTIÓN - GALIDAD

\title{
EVALUACIÓN NUTRICIONAL Y SOPORTE NUTRICIONAL POR VIA ENTERAL: NIVEL DE CONOCIMIENTOS DE ENFERMEROS DE UNIDADES DE TERAPIA INTENSIVA ADULTOS DE LOS HOSPITALES DE LA CIUDAD AUTÓNOMA DE BUENOS AIRES
}

\begin{abstract}
NUTRITIONAL SUPPORT AND EVALUATION BY ENTERAL ROUTE: NURSES' KNOWLEDGE LEVEL ABOUT ADULTS IN INTENSIVE THERAPY UNITS IN HOSPITALS FROM THE CITY OF BUENOS AIRES, ARGENTINA
\end{abstract}

\author{
*llari, S., **Salas, MA. \\ *Lcda. en Enfermería. Enfermera Soporte Nutricional. Hospital Italiano de Buenos Aires. ${ }^{* *}$ Lcdo. en \\ Enfermería. Egresado de la Universidad Austral. Buenos Aires. Argentina.
}

Palabras clave: Nutrición enteral, conocimientos de enfermería, sistema de salud.

Keywords: Enteral Nutrition, Nurses Knowledge, Health System.

\section{RESUMEN}

Desde principio de la década del '70 se publican estudios que demuestran las ventajas de equipos de soporte nutricional para implementar la Nutrición Enteral (NE) de manera eficiente en los pacientes que lo requieran, además la seguridad en la atención de la salud es un aspecto prioritario en la organización y funcionamiento del sistema de salud y posee implicancias directas en la calidad de atención de personas. En tal sentido la capacitación de enfermería es un factor de relevancia para la ejecución de cuidados en general y para el Soporte Nutricional (SN) en particular. Para que el SN sea efectivo y seguro son necesarios conocimientos sobre Evaluación Nutricional (EN), indicación, manejo de técnicas específicas, implementación, monitoreo y posibles complicaciones de la NE

\section{ABSTRACT}

Since the beginning of the 70s, studies have been published showing the benefits of teams of nutritional support to implement the Enteral Nutrition (EC) in an efficient way in patients that need it. Additionally, health care is a very important aspect in the management and running of the health system and it has direct implications in the quality of patients care. For this reason, nursing training is a relevant factor for the execution of general care and particularly for Nutritional Support. For Nutritional Support to be efficient and safe knowledge about Nutritional Evaluation, medical indications, management of specific techniques, implementation, monitoring and possible complications of Enteral Nutrition is needed. 


\section{INTRODUCCIÓN}

La Nutrición Enteral (NE) en los pacientes críticos ha cobrado gran relevancia en la última década, numerosas son las publicaciones en donde se enumeran los beneficios de una alimentación precoz en esta población en particular, sin embargo, contemporáneamente hay otro gran porcentaje en las cuales se relatan las complicaciones, muchas de ellas prevenibles por parte de enfermería si se poseen destrezas específicas sobre el tema. Esta fue la inquietud que nos hizo reflexionar sobre la necesidad de contar con personal entrenado para llevar a cabo los cuidados inherentes a la NE. En tal sentido decidimos desarrollar nuestra investigación indagando sobre formación de aquellos profesionales que desarrollan su actividad en las Unidades de Terapia Intensiva (UTI) de hospitales de la Ciudad de Buenos Aires.

El tema surge porque en el paciente críticamente enfermo la ingesta oral está seriamente comprometida debido a diversos factores que alteran la deglución en forma transitoria debido a la presencia del tubo endotraqueal o por patologías que contraindican el uso del aparato digestivo, por lo cual la implementación de algún tipo de soporte nutricional es fundamental para aportar los requerimientos calóricos y proteicos específicos para cada paciente.

Si el aparato digestivo conserva la motilidad gastrointestinal y no existen contraindicaciones para la administración de alimento por vía enteral, en la mayoría de los casos se utiliza un sondaje gástrico o postpilórico en aquellos pacientes que sufran de hipomotilidad gástrica 0 gastroparesia.

El éxito y la seguridad de la nutrición dependen en gran medida de la atención adecuada y la observación de la evolución por parte del personal de enfermería, ya que son quienes implementan la indicación de soporte nutricional y evalúan la tolerancia ${ }^{(1) .}$

En tal sentido, la capacitación de enfermería es un factor de relevancia para la ejecución de cuidados en general y para el soporte nutricional (SN) en particular. Para que el SN sea efectivo y seguro son necesarios conocimientos sobre Evaluación Nutricional (EN), indicación, manejo de técnicas específicas, implementación, monitoreo y posibles complicaciones de la NE ${ }^{(2)}$ dado que la posibilidad de escasa capacitación en este tema específicamente, podría promover intervenciones educativas a efectos de mejorar la calidad de atención nutricional.

Sin embargo, en las áreas críticas es frecuente observar falencias en la aplicación de las normas y recomendaciones para el seguimiento y control de la NE, lo que aumenta el riesgo de complicaciones. Numerosos factores influyen en estas circunstancias tales como la falta de sistematización de los cuidados, déficit de personal, escasa capacitación y también las carencias institucionales y de la infraestructura de un servicio de soporte nutricional y metabólico bien organizado para el monitoreo del soporte nutricional.

Las investigaciones sobre el soporte nutricional en el paciente crítico recomiendan la incorporación precoz de la alimentación, teniendo en cuenta que la malnutrición en el paciente crítico se asocia con un incremento de complicaciones y aumento de tasas de morbimortalidad y prolongada la estadía hospitalaria ${ }^{(3)}$. En un metanálisis realizado por Zaloga y Marik, que incorpora las publicaciones al respecto desde 1966 hasta 2000, se analizaron 15 trabajos prospectivos, aleatorizados y controlados tomando el concepto de NE precoz iniciada a las 36 horas de la lesión y se encontró que esta se asoció con una 
prevalencia significativamente menor de complicaciones infecciosas y con una reducción de la estadía hospitalaria de 2,2 días ${ }^{(4)}$.

Algunos autores sostienen que es oportuno iniciar la NE en las primeras 48 horas en los pacientes graves ya que son amplios los efectos beneficiosos y escasas las complicaciones si se las compara a la Nutrición Parenteral ${ }^{(5)}$, excepto en enfermos hemodinámicamente inestables.

Una investigación realizada en el hospital de clínicas de la ciudad Autónoma de Buenos Aires, evalúa el cumplimiento por parte del personal de enfermería de las normas de manipulación y administración de NE, encontrando que las mayores dificultades se relacionaron con la velocidad inadecuada en la administración, déficit en la administración de las fórmulas y escasa importancia asignada al control de la postura del paciente durante la infusión de la alimentación, sugiriendo la necesidad de implementar programas de educación continua, para promover el cuidado holístico y adecuado del paciente con soporte nutricional (6).

En un estudio prospectivo realizado en España en 160 pacientes relacionado a factores pronósticos de morbi-mortalidad en la NE se observó que sólo la cuarta parte recibía el soporte nutricional acorde a las recomendaciones nutricionales aconsejadas por las guías de procedimientos y actuación ${ }^{(7)}$.

\section{REFERENCIA TEÓRICA}

La alimentación por vía oral es la forma fisiológica de llevar a cabo la nutrición, no obstante, existen situaciones en la práctica clínica en que esto no es posible y se debe recurrir a formas alternativas a través de un soporte nutricional acorde a la situación específica de cada paciente.

Existe un alto porcentaje de pacientes que presentan algún grado de desnutrición al momento de su ingreso al hospital ${ }^{(28), ~ l a ~ d e s n u t r i c i o ́ n ~ t a m b i e ́ n ~ p u e d e ~ d e s a r r o l l a r s e ~ d u r a n t e ~ e l ~}$ curso de la hospitalización y con frecuencia se acentúa con la enfermedad e inclusive con algunos tratamientos médicos ${ }^{(8,9)}$. La desnutrición calórico-proteica (DCP) en cualquier situación clínica se debe a uno o varios de los siguientes factores patogénicos:

$>$ Ingesta nutricional insuficiente o inadecuada.

- Alteraciones de la digesto-absorción o del metabolismo de los nutrientes

$>$ Aumento de los requerimientos nutricionales.

$>$ Hiporexia o no colaboración (del personal o familiares) en el momento de la ingesta oral

La DCP es la deficiencia nutricional observada con más frecuencia en los pacientes hospitalizados denominada "desnutrición hospitalaria", la cual se caracteriza por una disminución de las reservas calórico-proteicas del organismo y por varias alteraciones de la función de órganos o sistemas debido a depleción proteica ${ }^{(10,11)}$.

En general la pérdida de 30 al 40 \% de la proteína corporal es causa de muerte.

La mayoría de las funciones fisiológicas se alteran con la pérdida de más del $20 \%$ de la proteína corporal lo cual aumenta las complicaciones postoperatorias y el tiempo de internación $(11,12,13)$. Si la depleción es rápida (por hipercatabolismo severo) una pérdida menor del $20 \%$ puede ser clínicamente significativa. 
El término soporte nutricional se refiere al proceso por el cual se trata de prevenir o retardar la aparición de una depleción proteica o de replecionar la proteína corporal si ya estuviera presente en el curso de la enfermedad (desnutrición secundaria) o durante la hospitalización (desnutrición hospitalaria) $^{(9)}$.

Las necesidades nutricionales de un paciente críticamente enfermo dependen de la gravedad de la lesión o de la enfermedad y del estado nutricional previo.

La respuesta hipermetabólica a la injuria puede modificar sustancialmente la homeostasis metabólica, hormonal, inmunológica y nutricional ${ }^{(11)}$.

La masa proteica de un adulto sano que ingiere una dieta normal se mantiene constante en un estado de equilibrio dinámico entre la síntesis y la degradación proteica. En condiciones normales, una dieta hiperproteica no incrementa la masa proteica, la cual solo se incrementa en los niños durante el crecimiento, en los deportistas y durante la recuperación de una DCP previa.

No existen depósitos de proteínas para la provisión de energía. Por lo tanto, cuando la ingesta calórico-proteica es menor que la necesaria para mantener el equilibrio entre la síntesis y la degradación se produce un balance negativo de proteínas lo que se acompaña de algún grado de deterioro funcional.

Durante las primeras 24 hs de ayuno la glucosa proviene del glucógeno hepático, luego a partir de la gluconeogénesis, es decir de la síntesis a partir de precursores como el ácido láctico, pirúvico, el glicerol y especialmente de los aminoácidos glucogénicos que provienen del catabolismo proteico.

De esta forma, en las primeras 24 horas de ayuno, un adulto normal de $70 \mathrm{Kg}$. degrada aproximadamente $75 \mathrm{gr}$ de proteína. Si se mantuviera este nivel de degradación proteica, es decir, si el organismo no se adaptara para ahorrar energía, en 25 a 30 días se perdería el 30 al $40 \%$ de la masa proteica lo cual conduciría a una disfunción multiorgánica y riesgo de muerte. La disminución del gasto energético y la utilización de combustibles alternativos son dos de los mecanismos mediante los cuales al disminuir la necesidad de aminoácidos glucogénicos disminuye la degradación proteica a 20 gr por día. Este ahorro de proteínas prolonga la supervivencia en caso de ayuno prolongado.

En el paciente críticamente enfermo la intensidad del trauma o de la infección determinan las demandas metabólicas. Existe una relación directa entre la gravedad de la lesión y el gasto energético total ${ }^{(16,17)}$.

La primera respuesta ante un traumatismo, injuria, o infección grave se denomina fase $E B B$, de shock o de bajo flujo y está caracterizada por disminución del consumo de oxigeno, del gasto energético y de la temperatura corporal; aumento de la glucemia, ácido láctico y de ácidos grasos libres. Los objetivos terapéuticos de esta etapa están dirigidos a la recuperación de las funciones vitales (mejorar el transporte y el consumo de oxígeno). Históricamente no se sugiere el soporte nutricional en esta etapa y hasta puede ser iatrogénico dado que el organismo no utiliza adecuadamente los sustratos exógenos, sin embargo en la actualidad es un tema controvertido.

Con la normalización del consumo de oxígeno se inicia la segunda fase denominada de alto flujo o Flow, hipermetabólica o hipercatabólica la cual se caracteriza por aumento del 
consumo de oxigeno, de la temperatura corporal, del volumen minuto cardiaco y de la frecuencia respiratoria y cardiaca.

\section{VALORACIÓN DEL ESTADO NUTRICIONAL DEL PACIENTE CRÍTICAMENTE ENFERMO}

La información sobre el estado nutricional de un paciente puede lograrse a través de su historia médica, social y alimentaria, el examen físico, sus medidas antropométricas y los análisis bioquímicos.

Algunos de ellos deben considerarse cuidadosamente en el paciente crítico ${ }^{(18,19,20)}$.

El peso es un índice del estado nutricional importante y necesario, incluso como punto de referencia de la nutrición, de las dosis de medicamentos que debe recibir un enfermo y de los parámetros de ventilación mecánica, pero no es un parámetro confiable en enfermos críticos.

El índice de masa corporal (IMC) valora la masa magra y la grasa corporal y se obtiene a partir de dividir el peso (en Kg.) por la talla al cuadrado (en Metros). Se puede concluir que la historia clínica del paciente debe incluir el registro del peso habitual, actual, el porcentaje de pérdida de peso y el IMC.

\section{Parámetros Bioquímicos:}

Creatinina: su excreción en orina es proporcional al contenido de creatina muscular y a la masa muscular corporal. Un gramo de creatinina en orina representa de 18 a $20 \mathrm{Kg}$. de músculo esquelético. El "índice creatinina-altura" determina la relación entre la eliminación de creatinina observada y la esperada para un adulto de igual edad y altura. Ello permitiría estimar la masa muscular esquelética según un valor estándar en función de la talla. La excreción de creatinina urinaria puede verse afectada por la edad, dieta, ejercicio, estrés y enfermedad renal. Además, para obtener un valor de fiabilidad son necesarias 3 determinaciones seriadas de orina de 24 horas y el cálculo de su promedio.

Balance Nitrogenado (BN): refleja si hay pérdida o ganancia de proteína tisular. El BN puede utilizarse con frecuencia para valorar la eficacia del tratamiento nutricional debido a que es fácil de realizar y tiene bajo costo.

El BN puede ser estimado por medio de una fórmula que considera que la excreción de nitrógeno es predominantemente a través de la orina como nitrógeno ureico urinario (NUU).

Proteínas plasmáticas: La albúmina, transferrina y prealbúmina son clásicamente usadas como marcadores de malnutrición, sin embargo presentan serias limitaciones, por ejemplo, la hidratación puede motivar cambios importantes en sus concentraciones plasmáticas independientemente de la velocidad de síntesis de las mismas y distintas enfermedades (hepática, síndrome nefrótico, eclampsia, enteropatías perdedoras de proteínas, etc.) son causas no nutricionales de disminución en su síntesis y concentración plasmática. La mayoría de estos parámetros, en el ámbito de la terapia intensiva, son inespecíficos.

Medidas Antropométricas: Se incluyen la medición de Pliegues Cutáneos y Circunferencia del brazo. 
Los principales errores en la interpretación de estas pruebas antropométricas son por IMPRECISION, ya que los resultados dependen mucho de quién mide, dónde y cómo se mide, POCA FIABILIDAD, pues factores como la hidratación, tono muscular, edad, influencian los resultados e INEXACTITUD, por haber muchas variables, en el pliegue por ejemplo, la compresión, el tejido adiposo y la piel.

Las medidas antropométricas no son útiles en los pacientes de Terapia Intensiva debido sobre todo a los cambios rápidos que se producen durante la hidratación y al decúbito.

Valoración Global Subjetiva (VGS): La VGS es un instrumento valioso para ser aplicado en estas circunstancias porque considera los hábitos alimenticios, pérdida de peso reciente o crónico, así como también otras condiciones del paciente que pueden obtenerse del interrogatorio. Los dos componentes básicos de este sistema son la entrevista clínica y el examen físico. Ninguno de los dos requiere equipos o procedimientos complicados. Se trata de una valoración fácil de realizar, económica y que ha demostrado utilidad ${ }^{(21)}$.

\section{Los objetivos de la valoración nutricional son:}

$>$ Identificar a los enfermos que puedan beneficiarse con la terapia nutricional para mantener o restaurar su estado nutricional.

> Ayudar a la identificación de la terapia nutricional más apropiada.

$>$ Valorar la eficacia de la terapia nutricional por medio del monitoreo rutinario del estado nutricional.

\section{EVALUACIÓN DE LAS NECESIDADES NUTRICIONALES: OBJETIVOS}

Cuando en un paciente se prevé que no puede obtener sus necesidades nutricionales por vía oral durante más de siete días, debe considerarse la necesidad de una NE y/o parenteral $(16,17,18)$. Los pacientes con déficit preexistente debido a diversas razones o por enfermedad deben iniciar el soporte nutricional lo antes posible o cuando se logre la estabilidad hemodinámica. Esta información la aportará la VGS.

La NE es la vía de elección cuando el tracto gastrointestinal está funcionante.

El objetivo de la terapia nutricional en el paciente críticamente enfermo es el sostén metabólico, no su repleción.

De hecho en esta situación, no existen condiciones que permitan metabolizar la cantidad total de calorías necesarias para reponer la deficiencia de energía gastada. La administración del aporte calórico completo puede ser dañina, ocasionando hiperglucemia, esteatosis hepática y otras complicaciones. Se debe considerar que tanto un aporte en exceso como en déficit pueden ser perjudiciales para el paciente. La sobrealimentación puede comprometer los efectos beneficiosos de la terapia nutricional.

\section{NUTRICIÓN ENTERAL (NE)}

Se denomina NE al conjunto de procesos terapéuticos mediante el cual se administran por vía digestiva nutrientes esenciales para mantener un adecuado estado nutricional, cuando la ingesta oral es imposible, no recomendado o insuficiente para cubrir los requerimientos basales. 
Esta vía de administración tiene una serie de ventajas fisiológicas, a las que se les debe sumar el hecho de ser una medida terapéutica accesible desde el punto de vista económico.

Entre las ventajas fisiológicas de la NE se destacan ${ }^{(19,20)}$.

- Previene la atrofia intestinal.

- Disminuye la translocación bacteriana: diferentes trabajos de investigación señalan que la NE mejora el efecto trófico de las vellosidades e incrementa la eficacia y eficiencia de las cilias. Además provoca una respuesta positiva en la inmunocompetencia del intestino delgado, especialmente en la referente a la $\lg \mathrm{A}$ secretora. Dadas sus propiedades antibacterianas y antivíricas, puede impedir la traslocación bacteriana a través de la mucosa intestinal.

- Disminuye las complicaciones infecciosas.

- Fortalece la función inmunitaria y facilita la regeneración de los tejidos ${ }^{(22) .}$

Este tipo de nutrición tiene una característica básica:

El empleo de sondas para su administración, dispositivos cilíndricos de diversos materiales, calibres y longitudes, que posee en el extremo proximal un conector y en el distal una punta roma con perforaciones, utilizada para administrar alimentos en sustitución de vía oral ${ }^{(23)}$.

\section{Rol de Enfermería en el monitoreo de la NE:}

Los cuidados que la enfermera va a brindar al paciente crítico con NE, serán el monitoreo, con el propósito de garantizar la correcta administración y la adecuación entre lo que se indica y lo que recibe. Es importante monitorizar permanentemente el volumen real infundido a cada paciente, porque en algunos casos el déficit de aporte puede ser tan importante como para convertirse en un mecanismo patogénico adicional en la depleción calóricoproteica de los pacientes críticos.

Asimismo es de suma importancia la prevención de complicaciones como vómitos, diarrea, constipación, alto residuo gástrico, salida accidental del acceso enteral y posición del paciente en el momento de la NE según ubicación de la sonda

\section{DISEÑO METODOLÓGICO DE LA INVESTIGACIÓN}

\section{Problema}

¿Cuál es el grado de capacitación y los conocimientos de los enfermeros que desarrollan su actividad en las Unidades de Terapia Intensiva (UTI) relacionado con el tema de la NE en pacientes críticos?

\section{Objetivo}

Describir el nivel de conocimiento que tienen los enfermeros de terapia intensiva acerca la NE en los pacientes críticos.

Tipo de estudio Descriptivo, cuantitativo, transversal, prospectivo. 


\section{Población}

Enfermeros que se desempeñan en atención directa en terapia intensiva de adultos en Hospitales de la Ciudad Autónoma de Buenos Aires, Argentina.

\section{Criterios de inclusión}

1. Auxiliares de Enfermería.

2. Enfermeros Profesionales o Universitarios.

3. Licenciados en Enfermería.

4. Tener al menos un año de ejercicio profesional.

5. Formar parte del personal de planta del Hospital.

6. Desempeñarse en la atención directa en el servicio de Terapia Intensiva de Adultos.

\section{Criterios de exclusión}

1. Todos los enfermeros que tengan a cargo pacientes con otro tipo de soporte nutricional.

2. No desear participar de la investigación.

3. No estar presente en le momento de recolección de datos.

\section{Criterios Temporo-Espaciales}

Enfermeros que se desempeñan en atención directa en los servicios de terapia intensiva de los hospitales de la Ciudad Autónoma de Buenos Aires durante el período de diciembre de 2007 a marzo de 2008.

\section{Recolección de datos}

La recolección de datos se ha realizado por medio de un cuestionario especialmente diseñado para la investigación. Se presentó el protocolo a la Dirección Médica y de Enfermería de cada Hospital seleccionado. Una vez obtenida la autorización y la nómina de enfermeros distribuidos por turnos de trabajo, se asistió a los servicios de Terapia Intensiva para tomar contacto con los enfermeros y explicar el carácter de la encuesta.

\section{Material y Métodos}

Se suministró un cuestionario escrito con preguntas abiertas y de respuestas múltiples a 70 enfermeros con más de 1 año de experiencia en UTI de Hospitales de la Ciudad Autónoma de Buenos Aires. El instrumento utilizado fue una encuesta voluntaria y anónima que consta de 3 dominios:

1) Formación profesional y actitud frente al soporte nutricional.

2) Evaluación nutricional y requerimientos calóricos.

3) Intervenciones de enfermería: conocimientos sobre indicación, técnicas especificas, implementación, monitoreo y prevención de complicaciones. 
El instrumento consta de un total de 31 ítems: los 4 primeros abordan variables sociodemográficas, que permitirán caracterizar a la muestra y eventualmente relacionarlas con la variable en estudio. Las 27 restantes son preguntas relacionadas a indicadores de la variable en estudio.

\section{Procesamiento de Datos}

Una vez recolectados los datos se volcaron en una planilla tipo Excel para su procesamiento. Se caracterizará la muestra a partir de las variables sociodemográficas; en el caso de variables cuantitativas se calculará la media y el desvío estándar; en las variables categóricas se calculará el porcentaje de cada categoría.

\section{Resultados:}

Se encuestaron 70 enfermeros (27\% licenciados, 47\% enfermeros universitarios, $11 \%$ enfermeros profesionales, $14 \%$ auxiliares de enfermería). Se analizaron los ítems ya descritos:

\section{Formación Profesional}

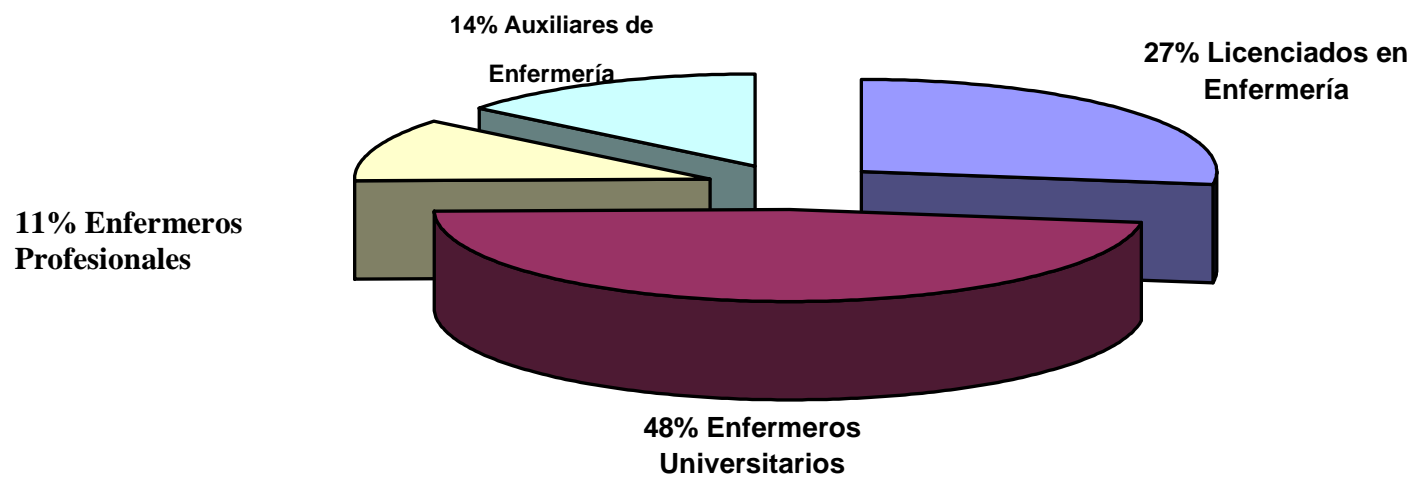

\section{Formación y actitud frente al SN:}

El 73\% (n:51) asumió que el adecuado tratamiento nutricional incide directamente en la morbi-mortalidad de los pacientes críticamente enfermos.

El 18,5\% (n:13) enfermeros realizaron algún curso de nutrición en los últimos 5 años.

El $20 \%$ leen regularmente artículos sobre SN. El $73 \%$ (n: 51$)$ consideró insuficiente su formación en nutrición. 


\section{Formación y Actitud frente al SN}

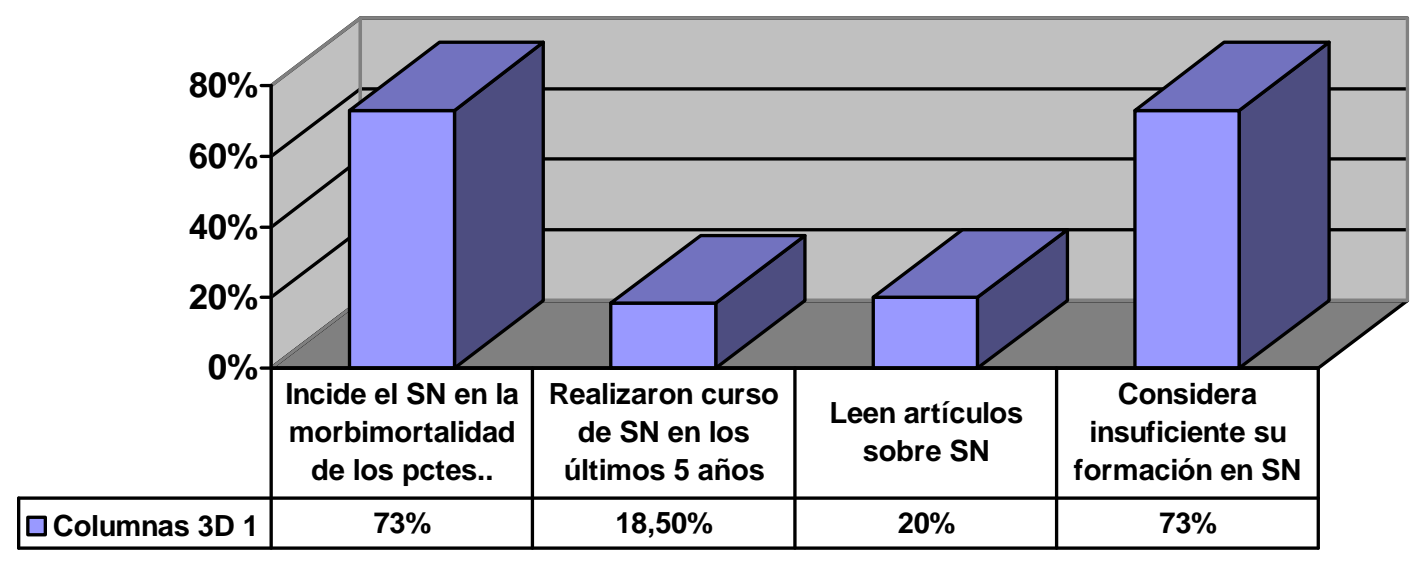

\section{Evaluación Nutricional:}

El 56\% (n: 39) desconocía la forma de realizar la valoración del estado nutricional. El51\% (n:36) considera que el inicio del soporte nutricional debe realizarse en las primeras 36 hs de internación. El 74\% (n: 52) no tenía conocimientos de como calcular los requerimientos calóricos y el 56\% (n:39) reconoce los beneficios de SN precoz en el paciente crítico. El 27\% $(\mathrm{n}: 19)$ conocen el rango normal del índice de masa corporal.

\section{Evaluación Nutricional}

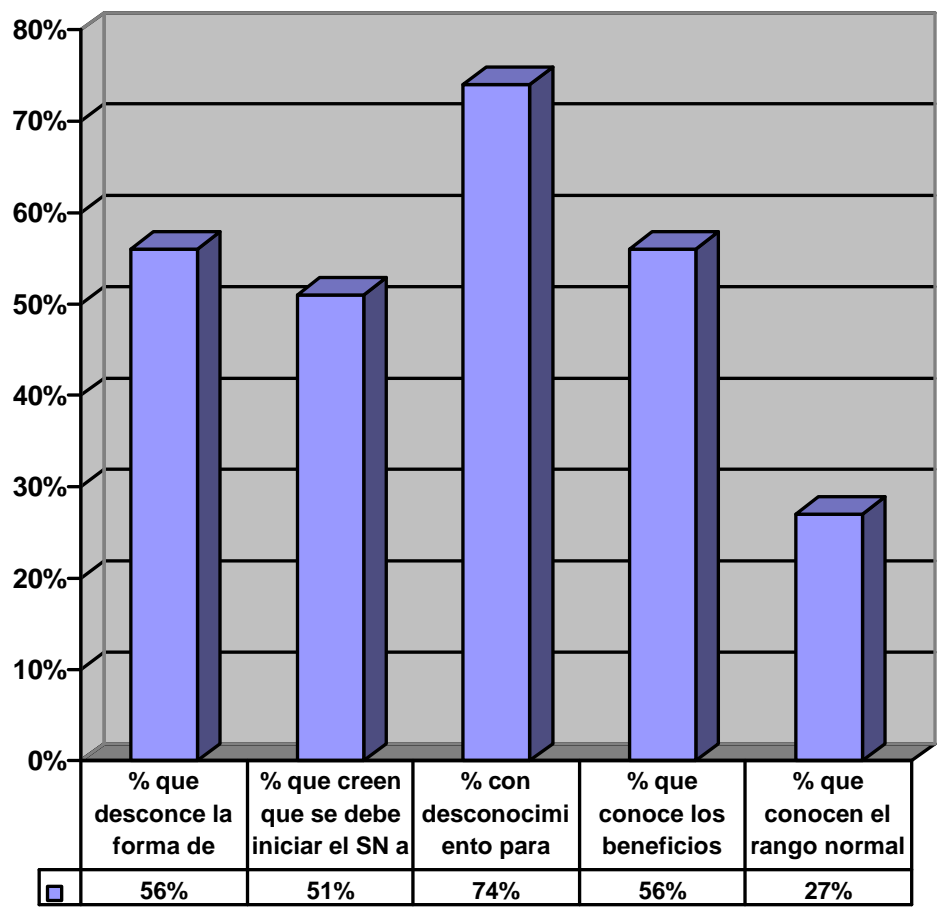




\section{Cuidados de enfermería}

El $47 \%$ (n: 33) contestó INCORRECTAMENTE a la pregunta referente a formas de administración del SN. El 58\% (n: 41) sabe cómo actuar frente al desplazamiento de la sonda de alimentación enteral. El 54\% (n: 38) desconocía la frecuencia del lavado de la sonda de alimentación y el 53\% (n: 37) No conocen la técnica de medición del Residuo Gástrico.

\section{Cuidados de enfermería}

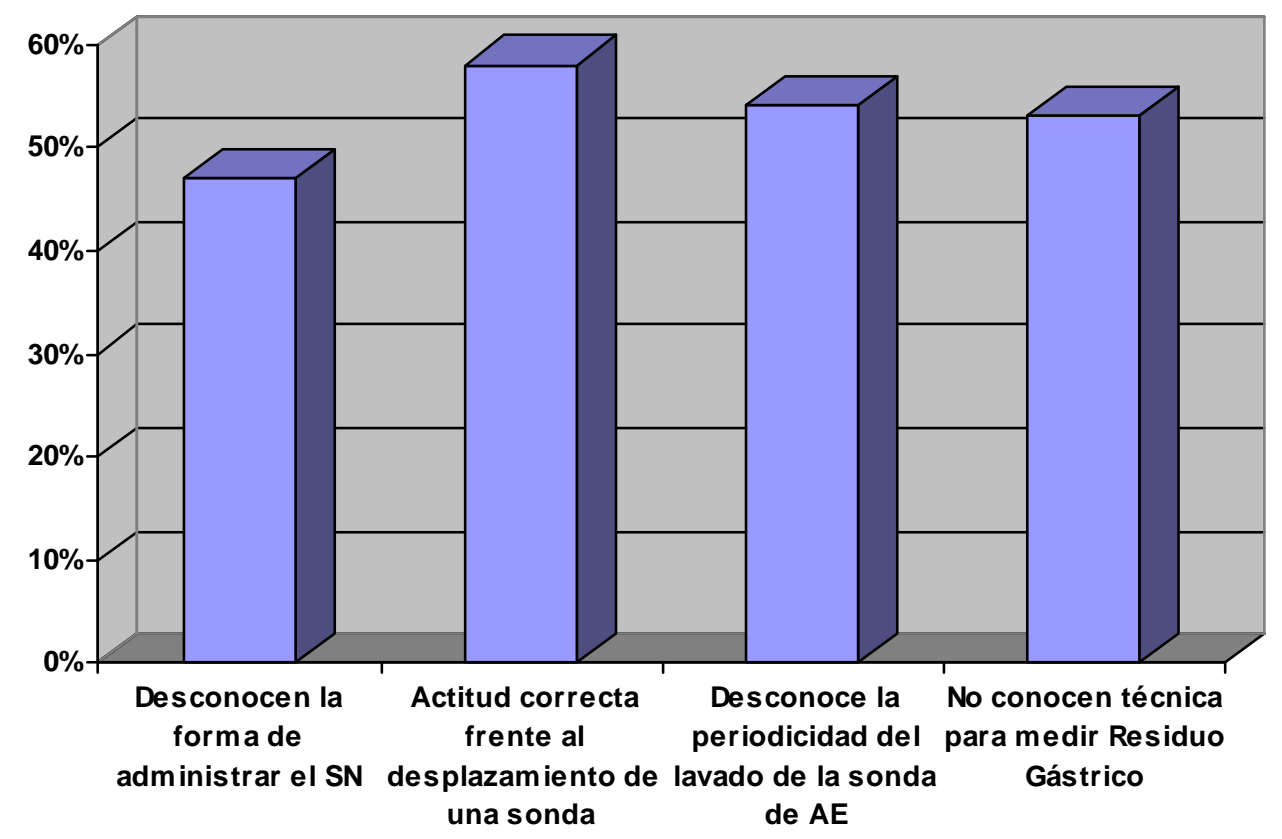

\section{Conclusiones}

Para el $73 \%$ de encuestados su formación y conocimientos sobre Soporte Nutricional (SN) son insuficientes. Poseen escasa instrucción en Evaluación Nutricional. Los cuidados de enfermería específicos fueron adecuados en un grupo menor. Considerando la elevada prevalencia de desnutrición hospitalaria y el riesgo nutricional de los enfermos críticos es preciso incorporar la asignatura de $\mathrm{SN}$ en la formación de grado como así también desarrollar cursos de postgrado que optimicen la capacitación de enfermería, incorporar técnicas e intervenciones específicas para mejorar la calidad de atención, adecuado monitoreo del paciente con soporte nutricional y la disminución de la morbimortalidad de los enfermos críticos.

\section{BIBLIOGRAFÍA}

1- Ilari S, Perman M. Terapia Intensiva: Implementación y Soporte Nutricional en Terapia Intensiva. $4^{\circ}$ ed. Buenos Aires: Medica Panamericana . 2005.

2- Delgado Sánchez M, Montana R, Rodríguez Cobos M, et al. La enfermera en la atención del paciente con soporte nutricional Hospital de San José de Bogota. [Internet]. Colombia: google.com.ar 2005. [Citado el 24 de mayo de 2006] disponible en http// www.bases.bireme.br/cgi-bin 
3- Castillo F.L, Romero P.C, MelladoT. P. Cuidados Intensivos Neurológicos: Santiago de Chile. Editorial Mediterráneo. 2004.

4- Marik P., Zaloga G. Early enteral nutrition in acutely ill patients: Asystematic review. Crit Care Med 2001;29:2264-70.

5- García Ávila, B. Y Grau T. La nutrición enteral precoz en el enfermo grave. [Internet]. España:Google.com.ar2005.[citado el 2 de octubre de 2006] disponible en http// www.scielo.isciii.es/scielo.php

6- Báez LM, Cavaciocchi M. Desempeño del personal de enfermería en la administración y manipuleo de alimentación enteral en pacientes internados [Internet].Buenos Aires, Argentina. Citado el 01/09/2006. Disponible en http// www.Nutrar com.htm

7- Moreno Pérez, O., Meoro Aviles, A., Martínez, A. et al. Factores pronósticos de morbimortalidad en nutrición hospitalaria: Estudio prospectivo. Nutrición Hospitalaria. 2005; 20 (3) ,210-216.

8- Mc Whirter JP, Pennington CR. Incidence and recognition of malnutrition in hospital. BMJ 1994; 308:945-948.

9- Waitzberg DL, Correia I, Echenique M. et al. Soporte Metabólico y terapia nutricional: Parte integral de la atención al paciente. Terapia Nutricional Total (TNT).2003.1-12.

10-Biolo G, Grimble G, Preiser JC, et al. Position paper of the ESICM Working Group Nutrition and Metabolism: Metabolic basis of nutrition in intensive care unit patients: ten critical questions. Intensive Care Med. 2002; 28: 1512-1520.

11- Cerra FB, Benitez MR, Blackburn GL, et al. Applied nutrition in ICU patients: a consensus statement of the American College of Chest Physisians. Chest 1997; 111: 769-778.

12- Heyland DK, Dhaliwal R, Drover JW, Gramlich L., Dodek P. And the Canadian Critical Care Clinical Practice Guidelines for Nutrition Support in Mechanically Ventilated, Critically Adult Patients. J Parenter Enteral Nutr. 2003; 27:355-373.

13-Heyland DK, Dhaliwal R, Day A, et al. Validation of the Canadian Clinical practice guidelines for nutrition Support in mechanically ventilated, critically ill adult patients: Results of a prospective observational study. Crit Care Med 2004; 32:2260-2266.

14- Barr J, Hecht M, Flavio KE, Khorama A and Gould MK. Outcomes in Critically ill Patients Before and After the Implementation of an Evidence-Based Nutritional Management Protocol. 2004; 125:1446-1457.

15- Martin CM, Doig GS, Heyland DK, et al. Southwestern Ontario Critical Care Research Network. Multicenter, cluster-randomized clinical trial of algorithms for critical care enteral and parenteral therapy (ACCEPT). Can Med Assoc J.2004;170:197-204.

16- Guidelines for the use parenteral and enteral nutrition in adult and pediatric patients. JPEN J Parenter Enteral Nutr.2002; 26:138.

17- Adam S, Bastón S. A study of problems associated with delivery of enteral feed in critically in patients in five ICU in the UK. Intensive Care Med. 1997;23: 261-266.

18- Zaloga GP. Early enteral nutrition improvises outcome: hypothesis or fact?. Crit Care Med. 1999; 27:259-261.

19-Barton, R. ¿Son benéficas en los pacientes críticamente enfermos las formulas enterales capaces de incrementar la respuesta inmune? Internet. Colombia: Google.com.ar. Citado el 13/06/06. Disponible en http// www.bases.bireme.br-cgi/bim.

20- Perman M. El soporte nutricional en el paciente critico. PROATI.

21-Goñi M, Yaffe M, Barreiro C, et al. Diagnostico de desnutrición y de riesgo de desnutrición en un Hospital General de Adultos. Lecturas sobre Nutrición.2000; 9 (2):68-72.

22- Barton R. Soporte nutricional en el paciente critico. [Internet]. Colombia: google.com.ar 2005. [Citado el 14 de julio de 2006]. Disponible en http// www.bases.bireme.br-cgi/bin

23-Asociación Argentina de Nutrición Enteral y Parenteral. Normas de buena practica clínica de soporte nutricional en pacientes adultos hospitalizados. [Internet].Argentina: 
google.com.ar 2006. [Citado el 10 de septiembre de 2006]. Disponible en http// www.aanep.com/index.php

24- Ilari S. Curso de actualización de soporte nutricional para Enfermería. Instituto Universitario y Departamento de Enfermería del Hospital Italiano de Buenos Aires. 2006:26-28.

25-Grupo de trabajo de Metabolismo y Nutrición de la Sociedad Española de Medicina Intensiva Coronaria y Crítica. Estudio multicentrico de incidencia de complicaciones de la nutrición enteral en el paciente grave: Estudio ICOMEP $2^{\circ}$ Parte. [Internet]. España 2005. [Citado el 15 agosto de 2006]. Disponible en http// www.scielo.isciii.es/scielo

26-Khers J, Olivares $\mathrm{O}$, et al. Normas para la preparación y administración de formulas enterales. [Internet]. Colombia: google.com.ar 2005. [Citado el 12 de julio de 2006]. Disponible en http// www.bases.bireme.br/cgi-bin

27- Ilari S. Alimentación Enteral. Revista de Enfermería del Hospital Italiano de Buenos Aires.2008. 2; (5): 25-29.

28- Estudio AANEP 99. Prevalencia de desnutrición en Hospitales de Argentina. Parte I: Metodología del estudio y análisis preliminar de resultados. Parte II Pronóstico Nutricional. www.aanep.com/index.php?s=educacion 